\title{
Characteristics of elite rhythmic gymnasts with scoliosis in Korea
}

\author{
Saejong Park, $\mathbf{P h D}^{\mathrm{a}}$, Donggyu Cho, $\mathrm{MD}^{\mathrm{b}}$, \& Lina Kim, $\mathbf{M D}^{\mathrm{c}^{*}}$ \\ ${ }^{a}$ Senior Researcher, Department of Sports Science, Korea Institute of Sports Science, Seoul, Korea \\ ${ }^{b}$ Department of Physical Medicine and Rehabilitation, Gangwon-do Rehabilitation Hostpital, \\ Gangwon-do, Republic of Korea \\ ${ }^{c}$ Medical director, Department of Physical Medicine and Rehabilitation, Sahmyook Medical Center, \\ Seoul, Republic of Korea
}

\begin{abstract}
The study aimed to investigate the demographic and physical characteristics of South Korean female elite rhythmic gymnasts with scoliosis. Twenty-eight female elite rhythmic gymnasts aged $16.1 \pm 3.0$ years were enrolled and divided by Cobb angle into a scoliosis group and a non-scoliosis group. In addition to the baseline characteristics of each group, visual analogue scale (VAS) scores for lumbago and results of the 36-item health survey version 2 (SF-36v2) questionnaires were collected. The pelvic tilt degree, leg length, hip range of motion and back-muscle strength were also measured and analyzed together. Radiographs of the thoracolumbar spine were taken for each subject. The scoliosis group $(n=17)$ had significantly higher age, height, weight, and body fat compared to the non-scoliosis group. Further, six of the scoliosis group gymnasts had lumbar compression fractures or spondylolysis. Spinal flexors and extensors of all the subjects were balanced in strength but the lateral flexors showed noticeable imbalance. Elite rhythmic gymnasts were found to be increasingly susceptible to scoliosis and other spinal disorders with age and years of training. Spinal lateral-flexor muscle imbalance was more severe with scoliosis.
\end{abstract}

Key words: Gymnastics, Scoliosis, Spinal curvature, Spinal injury, Sports injury

\section{Introduction}

Rhythmic gymnastics (RG) is a sport in which gymnasts perform body movements to music with or without apparatus: rope; hoop; ball; clubs; and ribbon (Sands, 2000). Rhythmic gymnasts continuously perform highly demanding athletic techniques within a limited time period (75 to 90 seconds) and space (13

Submitted : 15 June 2021

Revised : 30 November 2021

Accepted : 21 December 2021

Correspondence : magiclina@naver.com $\times 13$ meters). Thus, they require a high degree of agility, dexterity, strength, endurance, hand-eye coordination, and flexibility (FÉDÉRATION INTERNATIONALE DE GYMNASTIQUE, 2018).

Since flexibility, and particularly lumbar spine mobility, is considered one of the most important physical elements of the sport, gymnast scouting is mainly focused on younger age, shorter stature and thinner body type, and the training begins in childhood before puberty (Trexler et al., 2015). Chronic overtraining with dietary restriction is often considered 
mandatory for them, not only for the sake of athletic skills, but also for aesthetic reasons. Previous studies have revealed that rhythmic gymnasts are prone to growth retardation and chronic musculoskeletal injuries (Tanchev et al., 2000). Drinkwater et al emphasized that belated or incomplete management leads to chronic and/or severe injury (Drinkwater, 2000). Katz and Scerpella investigated the prevalence of lumbago in rhythmic gymnasts $(12 \%-85 \%)$ and its correlation to their age, athletic performance level, and training duration (Katz \& Scerpella, 2003). Pain in the back, ankles, and knees is common, but injuries are predominantly centered in the lumbar region.

In addition to musculoskeletal pain, scoliosis is prevalent in rhythmic gymnasts. Scoliosis is defined as a vertebral misalignment in the lateral plane with or without rotatory deformity (Negrini et al., 2018). The prevalence of scoliosis in the normal population is known to be $1-3 \%$, while that among rhythmic gymnasts is over 10 times greater (Tanchev et al., 2000). Scoliosis negatively affects body symmetry, and this leads to declines in affected athletes' performance, as well as low back pain. The seriousness of this problem has already been reported in many previous studies (Georgopoulos et al., 1999; Hutchinson, 1999; Tanchev et al., 2000; Trexler et al., 2015). As gymnasts begin their athletic careers at younger ages, general joint laxity, delayed maturity, and asymmetrical spinal loading have been demonstrated as three major risk factors for scoliosis (Tanchev et al., 2000).

Given the detrimental effect of scoliosis on elite rhythmic gymnasts who are mainly of prepubertal or pubertal age, it is important to clarify the etiology and set preventive strategies. To our best knowledge, however, there have been few studies dealing with elite rhythmic gymnasts worldwide and none in South Korea. Thus, this study aimed to investigate the demographic and physical characteristics of South Korean elite rhythmic gymnasts and pick out the specific factors interrelated with scoliosis. We expect that it would lay the groundwork for designing proper exercise regimens for them.

\section{Methods}

\section{Participants and grouping}

Participants were recruited from the population of South Korean elite rhythmic gymnasts who had won national competition awards. Those national team $(\mathrm{n}=$ 10) and backup team $(n=18)$ members were aged 16.1 \pm 3.0 years, and their average height, weight and training periods were $157.5 \pm 6.3 \mathrm{~cm}, 44.5 \pm 7.3 \mathrm{~kg}$, and $96.9 \pm$ 36.2 months each. Then they underwent diagnostic evaluation for scoliosis, physical examination, and fitness assessments of diverse parameters. The gymnasts with Cobb angles over $5^{\circ}$ were categorized in the scoliosis group (Figure 1). Their baseline characteristics and athletic performance level were analyzed in comparison with the others - the non-scoliosis group.

\section{Data collection}

To evaluate for the presence and subjective severity of low back pain, questionnaires were distributed to the participants. The short form 36-item health survey

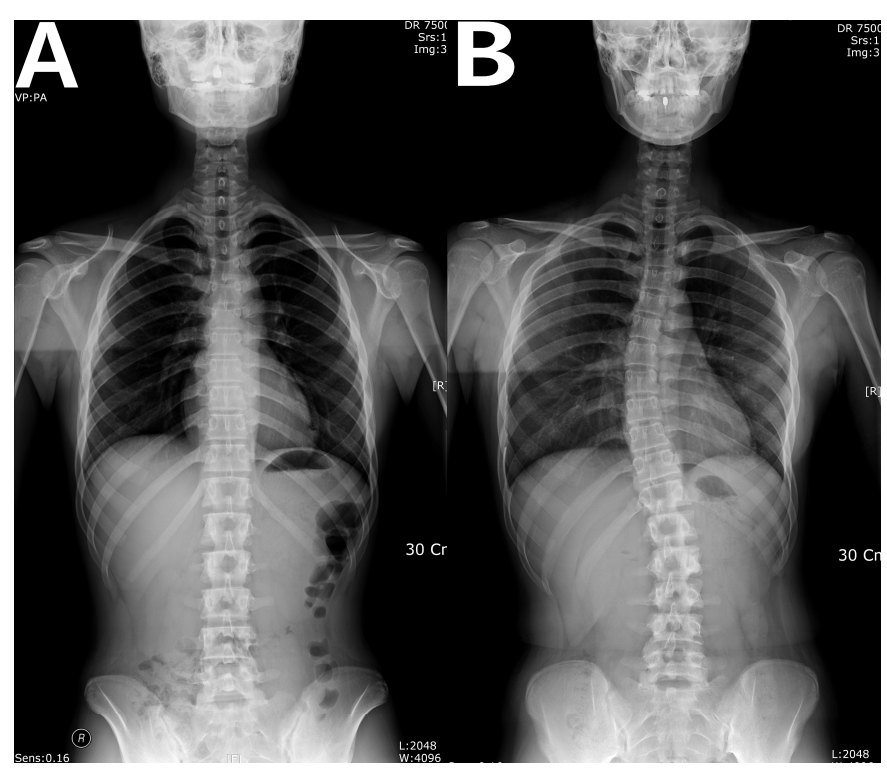

Figure 1. Whole spine posterior-anterior plain radiographic images of representative participants

While the Cobb angle of the normal group participant (A) was $0^{\circ}$, that of the scoliosis group participant (B) was $30.42^{\circ}$ 
version 2 (SF-36v2) was used to check their general health status (Lee Seung Ug \& Nam Bong Hyeon, 2003). Multiplanar neutral and stress (anterior-posterior, lateral and lateral flexion) plain radiographs of the entire vertebral column were taken.

Several physical metrics which may affect athletes' scoliosis were chosen and analyzed. Body composition was measured as an indirect indicator of physical maturity, and physical imbalance that could cause secondary scoliosis was differentiated by measuring leg length and pelvic tilt. Considering the characteristics of rhythmic gymnastics that require exceptional hip joint flexibility and core strength, hip joint range of motion and isometric lumbar muscle strength were also selected. Body fat (\%) and lean mass $(\mathrm{kg})$ were checked by using X-SCAN PLUS II (Jawon Medical Co., Kyungsan, Korea). Two specialists in physical medicine and rehabilitation measured the Cobb angle, the degree of pelvic tilt, and the active range of hip joint motion on each side using a standard hand-held goniometer (Sammons Preston Rolyan, Bolingbrook, IL, USA) and the average values were recorded. The bilateral lower limb lengths from the anterior superior iliac spine to the medial malleolus were evaluated in the same way to rule out secondary scoliosis arising from leg length discrepancy.

The maximal isometric strength of lumbar flexor, extensor, rotator, and lateral flexor muscles was evaluated twice using isometric testing machines (F110, F120, F130 and F150, David Health Solutions, Helsinki, Finland) and the highest result was recorded for each. Maximal isometric strength at the level of thoracolumbar column was estimated, for extension at 30 degrees, using F110 Lumbar/Thoracic Extension device, for flexion at 30 degrees, using David F130 Lumbar/Thoracic Flexion device, for lateral flexion at 0 degree, using David F150 Lumbar/Thoracic Lateral Flexion device and for lateral rotation (by moving the inferior limbs) at -30 degrees, using David F120 Lumbar/Thoracic Rotation device. Maximal isometric strength estimation, for all DAVID devices, at the level of lumbar-thoracic column, was estimated with hip and inferior limbs locked in a sitting position. Participants were instructed to execute all the movements (flexion, extension, lateral flexion and lateral rotation), at the level of thoracolumbar column, by developing a progressive isometric strength, avoiding sudden or uncontrollable movements (which could lead to erroneous values of maximal isometric force), for 5 seconds, maximal isometric strength being recorded in the last second of the test. Visual feedback and verbal stimulation were given during the testing.

\section{Data analysis}

Statistical analysis was performed using SPSS V.21.0 (IBM Corporation, USA). Averages and standard deviations of all evaluated parameters were calculated with statistical significance set at $p<0.05$. Wilcoxon signed-rank test was used to assess the differences in isometric hip joint ranges of motion, lumbar flexion-extension, and lumbar lateral-flexion strength. The relationship between athletic-career achievements and scoliosis was analyzed using Spearman's rho, and the difference in hip joint range of motion and lumbar isometric strength between the two groups was evaluated by Mann-Whitney $U$ test. The given values in the text are designated as the mean plus/minus standard deviation.

\section{Ethical considerations}

All participants provided written informed consent prior to enrollment, and the institutional review board of Korea Institute of Sports Science gave ethical approval (IRB number KISS-1001-001-01).

\section{Results}

Comparison of the scoliosis and non-scoliosis groups

Seventeen participants (61\%) were categorized in the scoliosis group and their Cobb angles ranged from $6.38^{\circ}$ to $30.42^{\circ}\left(13.97^{\circ} \pm 7.16^{\circ}\right)$. Compared to the non-scoliosis group, participants with scoliosis were 
significantly higher in age (17.0 \pm 3.0 versus $14.6 \pm 2.3$ years, $\mathrm{p}=0.036, \mathrm{~d}=0.88)$, height $(155.7 \pm 5.1$ versus $153.5 \pm 5.9 \mathrm{~cm}, \mathrm{p}=0.009, \mathrm{~d}=0.41)$, weight $(44.3 \pm 5.4$ versus $38.7 \pm 7.1 \mathrm{~kg}, \mathrm{p}=0.001, \mathrm{~d}=0.88)$ and body fat $(18.1 \% \pm 1.7 \%$ versus $13.9 \% \pm 3.1 \%, \mathrm{p}=0.000, \mathrm{~d}=$ 1.67). Total training duration was markedly longer in the scoliosis group $(102.9 \pm 39.0$ versus $87.5 \pm 30.8$ months, $\mathrm{p}=0.000, \mathrm{~d}=0.44$ ), but there was no significant correlation between training duration and Cobb angle $(\mathrm{p}=0.161)$.

Radiographic values and physical-examination results including hip flexibility measurements are shown in Table 1. There were significant differences between the two groups for Cobb angle $\left(13.97^{\circ} \pm 7.16^{\circ}\right.$ versus $0.00^{\circ}$ $\left.\pm 0.00^{\circ}, \mathrm{p}=0.000, \mathrm{~d}=2.76\right)$, back pain VAS $(4.29 \pm 1.90$ versus $0.45 \pm 1.04, p=0.000, d=2.51)$ and length of each lower extremity (right leg $89.41 \pm 3.55$ versus 86.00 $\pm 4.49 \mathrm{~cm}, \mathrm{p}=0.034, \mathrm{~d}=0.84$; left leg $89.47 \pm 3.61$ versus $85.91 \pm 4.57 \mathrm{~cm}, \mathrm{p}=0.030, \mathrm{~d}=0.86$ ). The overall hip-joint flexibility tended to be poorer in the scoliosis group, although only right hip extension showed a significant difference $\left(29.71^{\circ} \pm 11.11^{\circ}\right.$ versus $39.09^{\circ}$ $\pm 10.20^{\circ}, \mathrm{p}=0.033, \mathrm{~d}=0.88$ ).

There was no significant difference between the two groups for isometric strength of the lumbar muscles. When analyzed together, however, all the participants

Table 1. Radiographic values, physical examination results and isometric strength of lumbar muscles of non-scoliosis and scoliosis groups

\begin{tabular}{|c|c|c|c|c|}
\hline \multicolumn{2}{|c|}{ Variable } & Non-scoliosis group & Scoliosis group & p-value \\
\hline \multicolumn{2}{|l|}{ Cobb's angle $\left({ }^{\circ}\right)$} & $0.00 \pm 0.00$ & $13.97 \pm 7.16$ & $0.000^{*}$ \\
\hline \multicolumn{2}{|l|}{ SF-36v2(PCS) } & $52.00 \pm 2.00$ & $44.83 \pm 8.45$ & 0.172 \\
\hline \multicolumn{2}{|l|}{ SF-36v2(MCS) } & $44.67 \pm 7.51$ & $43.81 \pm 8.23$ & 0.870 \\
\hline \multicolumn{2}{|l|}{ Back pain (VAS) } & $0.45 \pm 1.04$ & $4.29 \pm 1.90$ & $0.000^{*}$ \\
\hline \multirow[t]{2}{*}{ Pelvic tilt $\left(^{\circ}\right)$} & Right & $0.00 \pm 0.00$ & $0.47 \pm 1.12$ & 0.104 \\
\hline & Left & $0.00 \pm 0.00$ & $1.82 \pm 4.10$ & 0.085 \\
\hline \multirow[t]{2}{*}{ Leg length (cm) } & Right & $86.00 \pm 4.49$ & $89.41 \pm 3.55$ & $0.034^{*}$ \\
\hline & Left & $85.91 \pm 4.57$ & $89.47 \pm 3.61$ & $0.030^{*}$ \\
\hline \multirow[t]{2}{*}{ Hip flexion $\left(^{\circ}\right)$} & Right & $142.27 \pm 12.32$ & $136.47 \pm 12.96$ & 0.249 \\
\hline & Left & $128.18 \pm 15.54$ & $119.41 \pm 15.90$ & 0.162 \\
\hline \multirow[t]{2}{*}{ Hip extension $\left(^{\circ}\right)$} & Right & $39.09 \pm 10.20$ & $29.71 \pm 11.11$ & $0.033^{*}$ \\
\hline & Left & $52.27 \pm 16.64$ & $43.82 \pm 16.25$ & 0.195 \\
\hline \multirow[t]{2}{*}{ Hip internal rotation $\left(^{\circ}\right)$} & Right & $33.64 \pm 6.74$ & $35.59 \pm 8.64$ & 0.532 \\
\hline & Left & $43.64 \pm 8.97$ & $40.00 \pm 10.90$ & 0.365 \\
\hline \multirow[t]{2}{*}{ Hip external rotation $\left(^{\circ}\right)$} & Right & $33.64 \pm 9.24$ & $28.24 \pm 9.83$ & 0.158 \\
\hline & Left & $25.00 \pm 4.47$ & $23.24 \pm 11.17$ & 0.624 \\
\hline \multirow[t]{2}{*}{ Hip abduction $\left(^{\circ}\right)$} & Right & $69.00 \pm 9.66$ & $63.82 \pm 9.28$ & 0.180 \\
\hline & Left & $77.50 \pm 17.68$ & $68.82 \pm 11.53$ & 0.134 \\
\hline \multicolumn{5}{|c|}{ Isometric lumbar muscle strength } \\
\hline \multicolumn{2}{|c|}{ Flexion $(\mathrm{Nm})$} & $59.10 \pm 18.92$ & $67.00 \pm 15.76$ & 0.150 \\
\hline \multicolumn{2}{|l|}{ Extension $(\mathrm{Nm})$} & $92.20 \pm 18.11$ & $99.10 \pm 17.02$ & 0.383 \\
\hline \multicolumn{2}{|c|}{ Right lateral flexion (Nm) } & $76.00 \pm 24.05$ & $79.00 \pm 20.12$ & 0.450 \\
\hline \multicolumn{2}{|c|}{ Left lateral flexion (Nm) } & $49.00 \pm 22.74$ & $52.20 \pm 15.00$ & 0.384 \\
\hline \multicolumn{2}{|l|}{ Right rotation $(\mathrm{Nm})$} & $46.20 \pm 22.11$ & $48.20 \pm 10.22$ & 0.650 \\
\hline \multicolumn{2}{|l|}{ Left rotation $(\mathrm{Nm})$} & $42.80 \pm 15.96$ & $50.00 \pm 8.52$ & 0.140 \\
\hline
\end{tabular}

Values are mean \pm SD unless otherwise stated

${ }^{*} \mathrm{p}<0.05$

SF-36v2, Short form 36-item health survey version 2; PCS, Physical component summary; MCS, Mental component summary; VAS, Visual analogue scale; Nm: Newton-meters 
Table 2. Isometric strength of lumbar muscles of 28 rhythmic gymnasts

\begin{tabular}{lccc}
\hline \hline & Right $\mathbf{( N m )}$ & Left (Nm) & \\
\hline Lateral flexor muscle & $77.50 \pm 21.63$ & $50.60 \pm 18.82$ & $0.000^{*}$ \\
Rotator muscle & $47.20 \pm 16.80$ & $46.40 \pm 12.99$ & 0.519 \\
\hline & Flexion (Nm) & Extension (Nm) & $0.000^{*}$ \\
\hline Lumbar & $63.05 \pm 17.42$ & $95.65 \pm 17.47$ & \\
Values are mean \pm SD unless otherwise stated & & & \\
N $<0.05$ & & & \\
Nm: Newton-meters & & &
\end{tabular}

showed lateral lumbar flexor strength imbalance (Table 2). In terms of dynamic standing balance, all the participants were graded 12 (most stable).

Other spinal disorders of scoliotic rhythmic gymnasts

In addition to diagnosing scoliosis, radiographic images were used to identify other spinal disorders in the rhythmic gymnasts. Among all 28 participants, 6 gymnasts presented skeletal abnormalities. In the non-scoliosis group, 1 was found to have anterior wedging of the twelfth thoracic and the first and second lumbar vertebral bodies. The other 5 with abnormalities were scoliotic participants diagnosed with compression fractures and 1 among them was accompanied with spondylolysis and spondylolisthesis (the fifth lumbar on the first sacral vertebra).

\section{Discussion}

This study targeted to investigate the demographic and physical characteristics of South Korean elite rhythmic gymnasts and clarify the specific factors interrelated with scoliosis. Through investigation, rhythmic gymnasts were found to be more vulnerable to spinal disorders including scoliosis and to be in poor subjective health status judging by their SF36-v2 scores. This tendency was found at a high rate in gymnasts with longer training period and a significant asymmetry in hip joint range of motion and lateral lumbar flexion was identified.
Previous studies focusing on RG were concentrated on the body composition, growth and development, nutrition, bone density and injuries of the gymnasts (Douda et al., 2002; Georgopoulos et al., 1999; Guidetti et al., 2000; Helge \& Kanstrup, 2002; Hutchinson, 1999; Klentrou, 2003; Sands, 2000; Theodoropoulou et al., 2005; Trexler et al., 2015). This research trend was based on the belief that physical traits such as a slender body shape and short stature positively affect these athletes' performance. To attain those advantages, restricted energy intake and high levels of physical training are usually started before puberty (Drinkwater, 2000). This prepubertal physical overloading and inadequate nutrition often leads to markedly delayed skeletal maturation and pubertal development (Georgopoulos et al., 1999). Not only overuse injuries of growth plates, but also endocrinological abnormalities such as low serum concentrations of sex steroids, decreased growth hormone secretion and disruption of insulin-like growth hormone homeostasis were reckoned to lead to young gymnasts' retardation in growth and pubertal development (Barron et al., 1985; Eliakim et al., 1996; Georgopoulos et al., 1999).

It was not long before sports medicine research directed its focus towards providing fundamental solutions for those problems. Researchers had previously focused on ways to improve the gymnasts' athletic performance by maximizing their atypical physical characteristics, not by treating them. Prior research has proven the correlation of certain physical factors with athletic performance; gymnasts were 
accordingly trained to enhance those abilities such as jumping strength and flexibility (Bordalo et al., 2015; Hutchinson, 1999). Throughout this training, however, many young gymnasts suffered repeated injuries and consequent chronic pain (Drinkwater, 2000). In South Korea, it was found that rhythmic gymnasts usually injured their back, ankles and knees; low-back injuries comprised $88.4 \%$ of the total documented pain focus (DB Lee et al., 1996). The cause of low-back pain varies, and may include heavy workload, repeated lifting and twisting, posture-induced stress, and inadequate core strength (among other factors) but scoliosis tends to be indicated as a key factor (Brotzman et al., 2011). More specifically, Tanchev et al. revealed that scoliosis was 10 times more common in rhythmic gymnasts than in normal adolescents and hypothesized 3 risk factors contributing to scoliosis in rhythmic gymnasts: hereditary joint laxity; delayed maturity due to overtraining; and chronic asymmetrical spinal loading (Tanchev et al., 2000).

The results of our study supported that hypothesis of Tanchev et al. The strength in Newton-meters (Nm) of right lumbar lateral flexor muscles and the degree of right hip flexion and left hip extension were significantly greater than that of the opposite side. Here, we need to consider the characteristic postures of RG as a sport. According to the point-scoring code, 'Body difficulty' and 'Balance' elements require a great degree of hip joint flexibility and one-legged standing balance (FÉDÉRATION INTERNATIONALE DE GYMNASTIQUE, 2018) (Figure 2). Thus, the majority of rhythmic gymnasts have trained a variety of postures and movements involving unilateral hip flexion. In other words, the gymnasts have been unintentionally obligated to aggravate body imbalances and distortions which could result in scoliosis.

The scoliosis group had significantly higher values for age, height, weight and body-fat percentage - to put it another way, they were physically more mature and developed - than the non-scoliosis group. Furthermore, $83 \%$ of the gymnasts who were diagnosed with comorbid skeletal abnormalities such as compression fractures and spondylolysis were found to be in the scoliosis group. A higher prevalence of scoliosis among the national team members $(70 \%)$ compared to that of backup members $(56 \%)$ also supported the hypothesis that long, intensive, and repetitive gymnastics training aggravates abnormal vertebral lateral curvature.

When it came to the comparison of hip joint flexibility and lumbar muscle isometric strength, no parameters except right hip extension displayed significant differences between the two groups. Greater asymmetry accompanying the scoliosis was expected, but this result could be attributed to the small sample size, and to the difference in basic characteristics of the participants in the two groups. In addition, all the participants scored 12 (most stable) in the dynamic body balance testing and this is probably due to their exceptional one-legged balance to grade with whole sole contact.

Since the study was performed on elite rhythmic gymnasts with a wide range of ages and the scoliosis

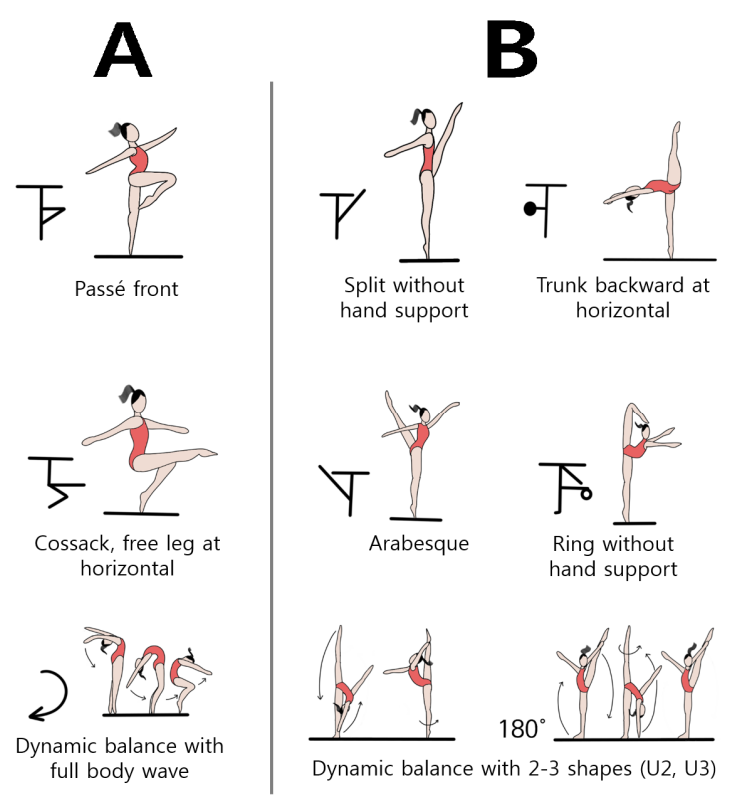

Figure 2. Types of "Balance Difficulty" according to the 2017-2020 CODE OF POINTS; Rhythmic Gymnastics

The values of the left column (A) are $0.1 \sim 0.2$ while those of the right column (B) are $0.4 \sim 0.6$. The higher the values are, the greater degree of one-legged split posture is required. 
group was significantly older than the non-scoliosis group, it is difficult to directly compare the basic and functional characteristics between the two groups. In addition, the results probably cannot be applied to amateur female rhythmic gymnasts considering the incomparably high training load and the small sample size of the elite participants. Nevertheless, those differences themselves reflect that continuous $R G$ training of young athletes would trigger and aggravate their spinal deformities and body imbalance as they age. Large-scale research would have been difficult due to the relatively small pool of rhythmic gymnasts in South Korea, with a total of 222 registered players (10 elite players) on December 31, 2018 (Korea Gymnastics Association, n.d.).

\section{Clinical implications}

A variety of scoliosis treatment options for the normal adolescent population such as exercise, bracing and surgery have been utilized worldwide. Exercise treatment includes hyperextension or hyperflexion exercises, trunk strengthening exercises, and muscle releasing and stretching (Negrini et al., 2018). However, there has been no clear consensus on appropriate scoliosis-preventing or correcting exercise for rhythmic gymnasts. Several studies applied core stability programs for the correction of scoliosis but those were only effective in improving balance ability (Borghuis et al., 2008). Ours is the first study we are aware of to systematically evaluate elite rhythmic gymnasts and further, to clarify the precise imbalances of lumbar lateral flexor muscles and hip joint flexibility among them. Therefore, we hope that our study results will pave the way for the development of appropriate exercise programs for $\mathrm{RG}$ athletes with scoliosis.

\section{Conclusion}

South Korean elite rhythmic gymnasts with scoliosis showed marked asymmetry of hip joint range of motion and lateral lumbar flexion strength when compared with non-scoliotic gymnasts. Furthermore, spinal disorders such as spondylolisthesis and compression fracture were more prevalent with the scoliotic gymnasts. As those trends were more pronounced with the their age and training experience, proper training and treatment methods focusing on the imbalance of lumbar lateral flexion power and hip range of motion is necessitated for their athletic achievements as well as their future quality of life.

\section{Acknowledgements}

This study was supported by a research grant from the Korea Sports Promotion Foundation.

\section{References}

Barron, J. L., Noakes, T. D., Levy, W., Smith, C., \& Millar, R. P. (1985). Hypothalamic Dysfunction in Overtrained Athletes. The Journal of Clinical Endocrinology \& Metabolism, 60(4), 803-806.

Bordalo, M. F., De Nazaré Portal, M., Cader, S., Perrotta, N. V., Dias Neto, J. M., \& Dantas, E. (2015). Comparison of the effect of two sports training methods on the flexibility of rhythmic gymnasts at different levels of biological maturation. The Journal of Sports Medicine and Physical Fitness, 55(5), 457-463.

Borghuis, J., Hof, A. L., \& Lemmink, K. A. P. M. (2008). The importance of sensory-motor control in providing core stability: Implications for measurement and training. Sports Medicine (Auckland, N.Z.), 38(11), 893-916. https://doi.org/ 10.2165/00007256-200838110-00002

Brotzman, S. B., Manske, R. C., \& Daugherty, K. (Eds.). (2011). Clinical orthopaedic rehabilitation: An evidence-based approach (3rd ed). Elsevier Mosby. DB Lee, YS Lee, \& YU An. (1996). Survey and Analysis of Sports Injuries and Treatment Patterns of Rhythmic Sportive Gymnastics Athletes. The 
Korean Journal of Sports Medicine, 14(2), 298-308.

Douda, H., Laparidis, K., \& Tokmakidis, S. (2002).

Long-Term training induces specific adaptations on the physique of rhythmic sports and female artistic gymnasts. European Journal of Sport Science, 2(3), $1-13$.

Drinkwater, B. L. (2000). Women in sport. Oxford; Malden, MA : Blackwell Science.

Eliakim, A., Brasel, J. A., Mohan, S., Barstow, T. J., Berman, N., \& Cooper, D. M. (1996). Physical fitness, endurance training, and the growth hormone-insulin-like growth factor I system in adolescent females. The Journal of Clinical Endocrinology \& Metabolism, 81(11), 3986-3992. FÉDÉRATION INTERNATIONALE DE GYMNASTIQUE. (2018). 2017 - 2020 CODE OF POINTS; Rhythmic Gymnastics. FÉDÉRATION INTERNATIONALE DE GYMNASTIQUE.

Georgopoulos, N., Markou, K., Theodoropoulou, A., Paraskevopoulou, P., Varaki, L., Kazantzi, Z., Leglise, M., \& Vagenakis, A. G. (1999). Growth and Pubertal Development in Elite Female Rhythmic Gymnasts. 84(12), 6.

Guidetti, L., Baldari, C., Capranica, L., Persichini, C., \& Figura, F. (2000). Energy Cost and Energy Sources of Ball Routine in Rhythmic Gymnasts. International Journal of Sports Medicine, 21(3), 205-209.

Helge, E. W., \& Kanstrup, I.-L. (2002). Bone density in female elite gymnasts: Impact of muscle strength and sex hormones: Medicine \& Science in Sports \& Exercise, 34(1), 174-180.

Hutchinson, M. R. (1999). Low back pain in elite rhythmic gymnasts. Medicine and Science in Sports and Exercise, 31(11), 1686-1688.

Katz, D. A., \& Scerpella, T. A. (2003). Anterior and Middle Column Thoracolumbar Spine Injuries in Young Female Gymnasts: Report of Seven Cases and Review of the Literature. The American Journal of Sports Medicine, 31(4), 611-616.
Klentrou, P. (2003). Onset of puberty, menstrual frequency, and body fat in elite rhythmic gymnasts compared with normal controls. British Journal of Sports Medicine, 37(6), 490-494.

Korea Gymnastics Association. (n.d.). Rhythmic gymnasts registration status of South Korea. http://www.gymnastics.or.kr/about/organization/na tionalteam/

Lee Seung Ug \& Nam Bong Hyeon. (2003). Testing the Validity of the Korean SF-36 Health Survey. Journal of Health Informatics and Statistics, 28(2), 3-24.

Negrini, S., Donzelli, S., Aulisa, A. G., Czaprowski, D., Schreiber, S., de Mauroy, J. C., Diers, H., Grivas, T. B., Knott, P., Kotwicki, T., Lebel, A., Marti, C., Maruyama, T., O’Brien, J., Price, N., Parent, E., Rigo, M., Romano, M., Stikeleather, L., ... Zaina, F. (2018). 2016 SOSORT guidelines: Orthopaedic and rehabilitation treatment of idiopathic scoliosis during growth. Scoliosis and Spinal Disorders, 13(1).

Sands, W. A. (2000). Injury prevention in women's gymnastics. Sports Medicine (Auckland, N.Z.), 30(5), 359-373.

Tanchev, P. I., Dzherov, A. D., Parushev, A. D., Dikov, D. M., \& Todorov, M. B. (2000). Scoliosis in Rhythmic Gymnasts: Spine, 25(11), 1367-1372.

Theodoropoulou, A., Markou, K. B., Vagenakis, G. A., Benardot, D., Leglise, M., Kourounis, G., Vagenakis, A. G., \& Georgopoulos, N. A. (2005). Delayed but Normally Progressed Puberty Is More Pronounced in Artistic Compared with Rhythmic Elite Gymnasts Due to the Intensity of Training. The Journal of Clinical Endocrinology \& Metabolism, 90(11), 6022-6027.

Trexler, E., Smith-Ryan, A., Roelofs, E., \& Hirsch, K. (2015). Body Composition, Muscle Quality and Scoliosis in Female Collegiate Gymnasts: A Pilot Study. International Journal of Sports Medicine, 36(13), 1087-1092. 